\title{
Excitatory Local Connections of Superficial Neurons in Rat Auditory Cortex
}

\author{
Dennis L. Barbour ${ }^{1}$ and Edward M. Callaway ${ }^{2}$ \\ ${ }^{1}$ Laboratory of Sensory Neuroscience and Neuroengineering, Washington University in St. Louis, St. Louis, Missouri 63130, and ${ }^{2}$ Systems Neurobiology \\ Laboratories, Salk Institute for Biological Studies, La Jolla, California 92037
}

\begin{abstract}
The mammalian cerebral cortex consists of multiple areas specialized for processing information for many different sensory modalities. Although the basic structure is similar for each cortical area, specialized neural connections likely mediate unique information processing requirements. Relative to primary visual (V1) and somatosensory (S1) cortices, little is known about the intrinsic connectivity of primary auditory cortex (A1). To better understand the flow of information from the thalamus to and through rat A1, we made use of a rapid, high-throughput screening method exploiting laser-induced uncaging of glutamate to construct excitatory input maps of individual neurons. We found that excitatory inputs to layer $2 / 3$ pyramidal neurons were similar to those in $\mathrm{V} 1$ and $\mathrm{S} 1$; these cells received strong excitation primarily from layers 2-4. Both anatomical and physiological observations, however, indicate that inputs and outputs of layer 4 excitatory neurons in $\mathrm{A} 1$ contrast with those in V1 and S1. Layer 2/3 pyramids in A1 have substantial axonal arbors in layer 4, and photostimulation demonstrates that these pyramids can connect to layer 4 excitatory neurons. Furthermore, most or all of these layer 4 excitatory neurons project out of the local cortical circuit. Unlike S1 and V1, where feedback to layer 4 is mediated exclusively by indirect local circuits involving layer $2 / 3$ projections to deep layers and deep feedback to layer 4 , layer 4 of A1 integrates thalamic and strong layer 4 recurrent excitatory input with relatively direct feedback from layer $2 / 3$ and provides direct cortical output.
\end{abstract}

Key words: auditory cortex; rat; lamina; column; glutamate; synapse

\section{Introduction}

The core areas of auditory cortex, including primary auditory cortex (A1), receive the greatest proportion of ascending projections arising from the auditory thalamus and represent the first auditory areas in the cortical hierarchy. Similarly to other primary sensory areas, local processing of this information in A1 is performed by cell types distinct in their dendritic morphologies, neurotransmitters, electrotonic properties, and axonal projections (Cruikshank et al., 2002; Winer et al., 2005). Thalamic input patterns into A1 of several species, however, as well as recipient cell types, appear to exhibit unique patterns relative to other primary sensory areas. In particular, thalamic projection density into A1 appears to be graded with cortical depth rather than abrupt at laminar boundaries, and most thalamic axonal terminals are concentrated fairly uniformly in layers 3 and 4 (Winer, 1984a; Metherate and Aramakis, 1999). The auditory thalamic nuclei that project to A1 also appear to grossly intermingle their projections with one another, although these projections are anatomically distinct on a microscopic level (Roger and Arnault, 1989; Huang and Winer, 2000). Furthermore, layer 2/3 pyramidal neurons within A1 appear to form synaptic connections with

\footnotetext{
Received May 7, 2008; revised Aug. 7, 2008; accepted Sept. 17, 2008.

This work was supported by National Institutes of Health Grants EY010742 (E.M.C.) and DC008880 (D.L.B.). We thank D. Larsen, J. McCoin, I. Acevedo, and J. D. Dickman for generous histology assistance.

Correspondence should be addressed to Dr. Dennis L. Barbour, Campus Box 1097, One Brookings Drive, Washington University in St. Louis, St. Louis, M0 63130. E-mail: dbarbour@biomed.wustl.edu. DOI:10.1523/JNEUROSCI.2093-08.2008

Copyright $\odot 2008$ Society for Neuroscience 0270-6474/08/2811174-12\$15.00/0
}

other pyramidal neurons that exhibit biophysical properties unique to auditory cortex (Atzori et al., 2001). Together, this evidence implies that A1 neurons may form functional circuits that are anatomically intertwined, functionally precise, and physiologically distinct from other primary sensory areas.

Cortical circuits can be precise at many different levels of organization. For example, within a population of anatomically indistinguishable layer 2/3 pyramidal neurons, distinct subpopulations can exhibit connectivity differences depending on their location relative to thalamic projections (Koralek et al., 1988; Kim and Ebner, 1999; Shepherd et al., 2003). Furthermore, any given cortical layer contains the dendritic processes of multiple cell types, and axons projecting to that region can selectively connect to some cell types relative to others (Callaway, 2002). The possibility exists, then, that specific microcircuits exist in A1 that are anatomically distinct but challenging to discern by classical neuroanatomical tracing techniques or cytological investigations. Elucidation of this local functional circuitry may aid in constructing biologically accurate computational models of the neural network underlying potentially unique auditory functions.

This study makes use of an established high-throughput technique to map the local excitatory circuitry among several subtypes of neurons in layers 2-4 of rat A1. Focal uncaging of glutamate by laser-scanning photostimulation allows rapid successive stimulation of small populations of neurons within small diffraction-limited volumes of an acute brain slice (Callaway and Katz, 1993; Katz and Dalva, 1994; Sawatari and Callaway, 2000; 
Shepherd et al., 2003; Jin et al., 2006). The resulting input maps are quantified and compared across neuron classes to determine local circuitry patterns among these auditory cortex neurons, and the results are discussed in the context of other sensory circuits in neocortex.

A brief report of these results has appeared previously in abstract form (Barbour and Callaway, 2004).

\section{Materials and Methods}

Physiological recordings. All animal procedures were approved by the Salk Institute Animal Care and Use Committee. Long-Evans rats with developmentally mature cortical neuron morphology (P25-P31) were given an anesthetic overdose (Nembutal, $35 \mathrm{mg} / \mathrm{kg}$ ). Their brains were then removed and immersed in ice-cold artificial cerebrospinal solution (ACSF; composition in mM: $124 \mathrm{NaCl}, 5 \mathrm{KCl}, 1.25 \mathrm{KH}_{2} \mathrm{PO}_{4}, 1.3 \mathrm{MgSO}_{4}$, $3.2 \mathrm{CaCl}_{2}, 26 \mathrm{NaHCO}_{3}$ and 10 glucose) for $1 \mathrm{~min}$. The hemispheres were then separated and the cerebellum and subcortical structures removed. A $5 \mathrm{~mm}$ block of brain was created by making parallel coronal cuts $\sim 2.5$ and $7.5 \mathrm{~mm}$ from the posterior margin of the cerebral cortex. This block was glued anterior side down to the stage of a vibrating tissue slicer (Oxford Vibratome; Oxford Instruments) and immersed in ice-cold ACSF. Brain slices, $400 \mu \mathrm{m}$ thick, were cut from this block in a posteriorto-anterior direction. Because the extent of auditory cortex has been shown to be coincident with that of the hippocampus (Doron et al., 2002; Rutkowski et al., 2003), only slices containing clearly defined hippocampus were saved for later experimentation ( $\sim 6-8$ per hemisphere).

The slices were incubated for at least one hour at $34^{\circ} \mathrm{C}$ in a custom air/fluid interface chamber infused with $95 \%$ oxygen, $5 \%$ carbon dioxide and ACSF. Slices were then placed onto a fixed-stage microscope (BX41; Olympus) and perfused with room-temperature ACSF containing 80-100 $\mu \mathrm{m} \gamma$-CNB caged glutamate (G-7055; Molecular Probes). Infrared-differential interference contrast (IR-DIC) optics were used to locate cells of interest and direct the placement of micropipette patch electrodes (6-10 $\mathrm{M} \Omega$ ) made in a Flaming/Brown micropipette puller (P-97; Sutter Instrument ). Cells were only isolated within the slice region having a visibly prominent layer 4 under IR-DIC optics, later confirmed with a cytochrome oxidase stain. Isolated cells were located $2.46 \pm 0.28 \mathrm{~mm}$ dorsal to the perirhinal fissure along the cortical surface, consistent with the location of auditory cortex determined from combined physiological and histological studies (Doron et al., 2002). Patch pipettes contained intracellular solution [ICS; (in mM) $130 \mathrm{~K}$-gluconate, $6 \mathrm{KCl}, 2 \mathrm{MgCl}_{2}, 0.2 \mathrm{EGTA}$, 10 HEPES, $2.5 \mathrm{Na}_{2}$ ATP, $0.5 \mathrm{Na}_{2} \mathrm{GTP}$, and $10 \mathrm{~K}$-phosphocreatine, and $0.3 \%$ biocytin; $\mathrm{pH}$ was adjusted to 7.25 with $\mathrm{KOH}$ and a $\mathrm{pH}$ meter (Corning 320); osmolarity was adjusted to 285-290 mOsm with sucrose and a vapor pressure osmometer (Wescore 5500)].

Cells were analyzed in whole-cell patch voltage-clamp mode and held at a potential of $-65 \mathrm{mV}$ (the reversal potential for $\mathrm{Cl}^{-}$). Ultraviolet light (50 $\mathrm{mW}$ at $355 \mathrm{~nm}$ ) from a diode-pumped, solid-state laser (Series 3500; DPSS Lasers) illuminated the slice through a $40 \times$ water-immersion objective (Olympus). Laser stimulations (duration, $10 \mathrm{~ms}$ ) systematically flashed each slice location in a grid with horizontal and vertical spacings of $50 \mu \mathrm{m}$. Adjacent sites were never stimulated more rapidly than $10 \mathrm{~s}$ apart. Inward currents occurring shortly after a laser flash indicate either a direct response (activation of glutamate receptors on the cell of interest) or the EPSC input from a monosynaptically connected cell (Callaway and Katz, 1993; Dantzker and Callaway, 2000; Shepherd et al., 2003). With subsequent analysis (see below), direct and synaptic events may be readily distinguished.

Anatomical and physiological analysis. Anatomical methods for cell labeling and laminar boundary determination were described in detail previously (Yabuta and Callaway, 1998; Dantzker and Callaway, 2000). Briefly, after each experiment, the brain slices were prepared for anatomical analysis by fixation in $4 \%$ paraformaldehyde followed by dehydration $24 \mathrm{~h}$ later in $30 \%$ sucrose. After another $24 \mathrm{~h}$, the fixed, dehydrated slices were then frozen and resectioned in $80 \mu \mathrm{m}$ increments on a freezing microtome (HM 400; Microm International). Sections were stained for cytochrome oxidase and biocytin to establish laminar borders and visualize the filled cell, respectively (Roger and Arnault, 1989; Wallace and Palmer, 2008). Borders and cells were drawn with Neurolucida software (MicroBrightfield). Verification that the angle of slice cutting was nearly parallel to major intraslice neuronal processes was performed during experimentation by observing under IR-DIC optics that the apical dendrites of deep pyramidal cells were intact to layer 1. Preservation of intraslice processes was also confirmed anatomically by observing the apical dendrites of filled infragranular pyramidal neurons and by observing descending axonal projections of filled layer supragranular pyramidal neurons. Stimulation sites were assigned to cortical layers by custom software written in Matlab (Mathworks) that aligned the stimulation and anatomical maps using registration markings made by the laser (Dantzker and Callaway, 2000).

Excitatory neurons in layers 2-4 were identified on the basis of their spiny dendritic trees. Cells that sent an apical process superficially to the layer $1 / 2$ border and tufted were considered pyramidal cells. Another cell type observed in layer 4 sent an apical process into layer 3 but not to the layer $1 / 2$ border and did not tuft. These cells are referred to as star pyramids. Inhibitory neurons in layers $2-4$ were determined anatomically by their spineless dendritic trees. Two electrophysiological categories of inhibitory interneurons were identified by their spiking behavior in response to $300 \mathrm{~ms}$ steps of depolarizing current: adapting and fast-spiking. Fast-spiking cells were distinguished from adapting cells by linear increases in spiking rate as a function of depolarizing current amplitude and an interspike interval ratio between the first and last pairs of spikes approaching unity (Connors et al., 1982; Connors and Gutnick, 1990; Agmon and Connors, 1992; Metherate and Aramakis, 1999). Cells with interspike interval ratios $>0.7$ were classified as fast-spiking $(0.89 \pm$ $0.12)$ and cells with ratios $<0.7$ were classified as adapting $(0.47 \pm 0.13)$. Both cell types exhibited smooth, aspiny dendrites with a variety of typically compact dendritic morphologies. Axonal projections were extensive and local with no axonal projections into the white matter.

Excitatory current maps were made by measuring inward charge transfer for each stimulation site (i.e., sum of EPSC areas). EPSCs were cataloged and quantified using MiniAnalysis software (Synaptosoft) within a temporal window of $150 \mathrm{~ms}$ after laser onset (i.e., the stimulation interval). Stimulation sites resulting in direct currents, as determined by short latency ( $<10 \mathrm{~ms}$ after laser onset), long duration (tens of milliseconds), and large amplitude relative to isolated synaptic currents, were excluded from analysis. Spontaneous events were also quantified with MiniAnalysis throughout a control interval of $150 \mathrm{~ms}$ beginning at least $400 \mathrm{~ms}$ after laser stimulation. The same total recording time was used both for control intervals containing only spontaneous events and for stimulation intervals containing spontaneous events and, potentially, stimulated events arising from glutamate-induced presynaptic neuronal activity. The experimenter cataloguing events was blinded to both the type of time interval (stimulation or control) and the stimulation site location.

Excitatory input maps for each cell were visualized as total charge transfer per stimulation site during the stimulation interval, and laminar summaries were calculated as the mean charge transfer across all stimulation sites within each layer. Comparing these laminar charge transfer values directly across cells is unwise because of inevitable variation in series resistance each time a neuron is patched. Consequently, two methods of evaluating laminar input on a cell-by-cell basis were devised. First, laminar charge transfer values were expressed in units of spontaneous charge transfer SD by converting to $Z$ scores:

$$
\mathrm{Z}=\frac{\overline{C T_{\text {stim }}}-\overline{C T_{\text {spont }}}}{\sigma_{\text {spont }}},
$$

where $\overline{C T_{\text {stim }}}$ represents the mean charge transfer elicited for sites within a given layer by stimulation, $\overline{C T_{\text {spont }}}$ represents the mean charge transfer for the cell during the unstimulated control interval ("spontaneous") and $\sigma_{\text {spont }}$ represents the sample SD of spontaneous charge transfer. This metric also removes the effect of spontaneous EPSCs on estimates of excitatory input and results in summary values depicting for a given cell class the magnitude of evoked excitatory input from each layer. 


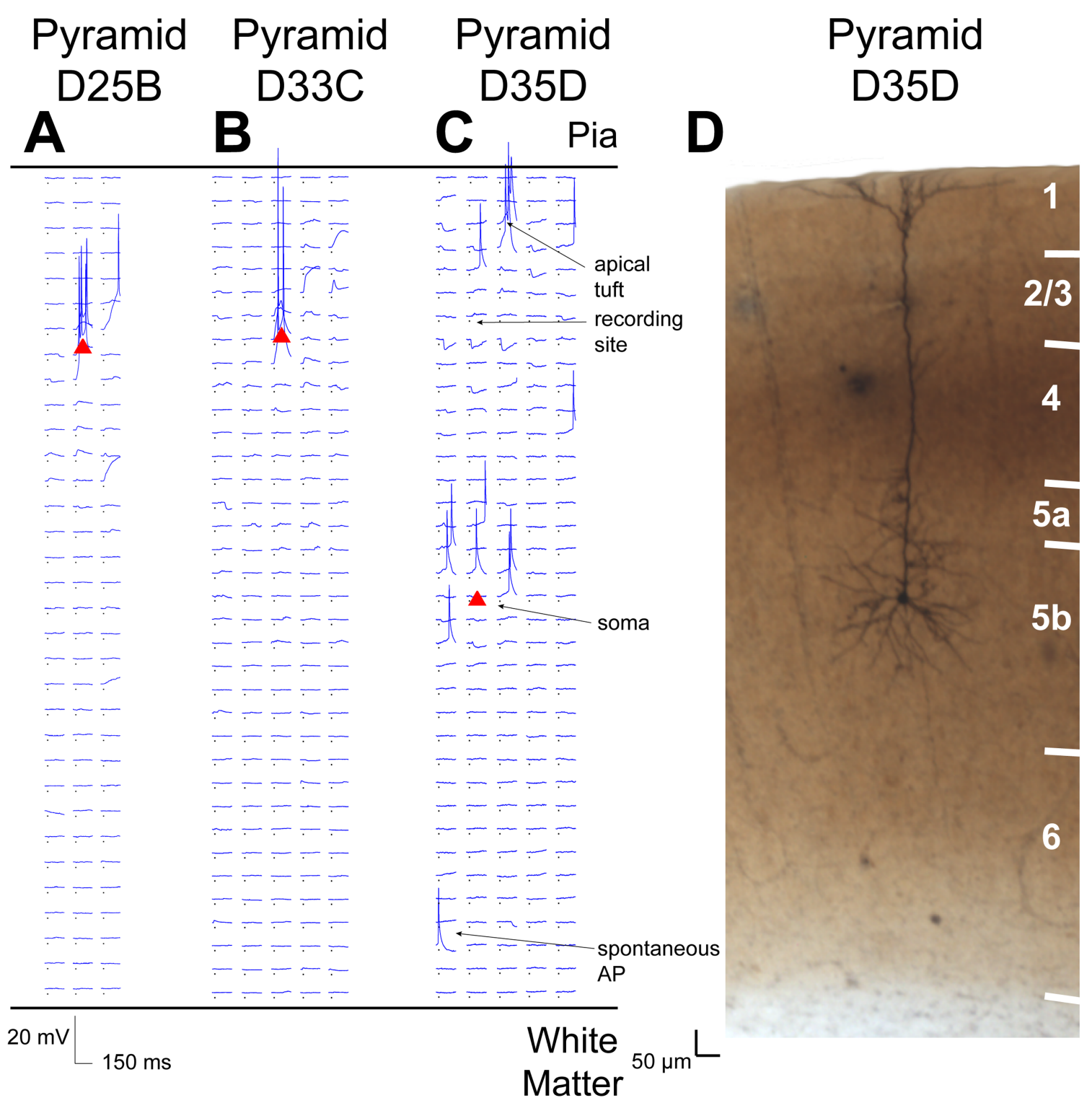

Figure 1. Spatial resolution of photostimulation technique. $A, B$, Voltage traces of layer $2 / 3$ pyramidal neurons held in whole-cell current clamp indicate that action potentials in a neuron generally occur only when that neuron is photostimulated near its soma. Soma locations of patched cells are indicated by red triangles. Voltage traces (blue) are plotted at the corresponding site of laser stimulation. Laser duration of $10 \mathrm{~ms}$ is indicated by a horizontal line at each voltage plot. Scale bars correspond to either the voltage traces or distance within the slice. $C$, Neurons with extensive tufting in layer 1 also fire action potentials when stimulated near their tufts. A layer 5 pyramidal neuron recorded from its apical dendrite demonstrates not only stimulated action potentials near its soma but also near its extensive apical tuft in layer 1. For this reason, stimulation in layer 1 is not considered when evaluating input maps. D, Apical dendrite, nearly $1 \mathrm{~mm}$ long of layer 5 pyramidal neuron, is preserved within a single $80 \mu \mathrm{m}$ section of the slice, indicating that intraslice projections are largely intact.

Second, for each cell, the mean stimulated laminar charge transfer values were evaluated for statistical significance relative to the mean spontaneous charge transfer by a repeated-measures ANOVA $(p<$ 0.05), post hoc corrected with a Tukey's honestly significant difference (HSD) test for multiple comparisons. To obtain a population estimate of laminar input patterns independent of the collective strength of that input, overall counts of cells receiving significant laminar input (determined by the ANOVA) were evaluated with a two-tailed binomial test. Neuron counts higher than expected from the binomial distribution with a success probability of 0.05 were considered significant. Trends in the relative proportion of excitatory input into cells from different layers were quantified with a linear regression and the significance evaluated with a regression $F$ test. Differences in laminar input strength between cell types were assessed using a Wilcoxon rank-sum test of $Z$ scores.

\section{Results}

Laser-scanning photostimulation resolution and neuronal process preservation

We recorded excitatory input maps from a total of 66 neurons in rat auditory cortex brain slices. Under the experimental conditions used for physiological recording, spontaneous neuronal spiking was rare, and laser photostimulation typically depolarized neurons held in whole-cell, current clamp mode above action potential threshold only for laser flashes near their somas (Fig. 1A-C). These results mirror previous findings under similar experimental conditions (Dantzker and Callaway, 2000; Shepherd et al., 2003; Yoshimura et al., 2005) and confirm that for the mapping experiments conducted under voltage clamp, inward currents after photostimulation are likely to reflect monosynap- 

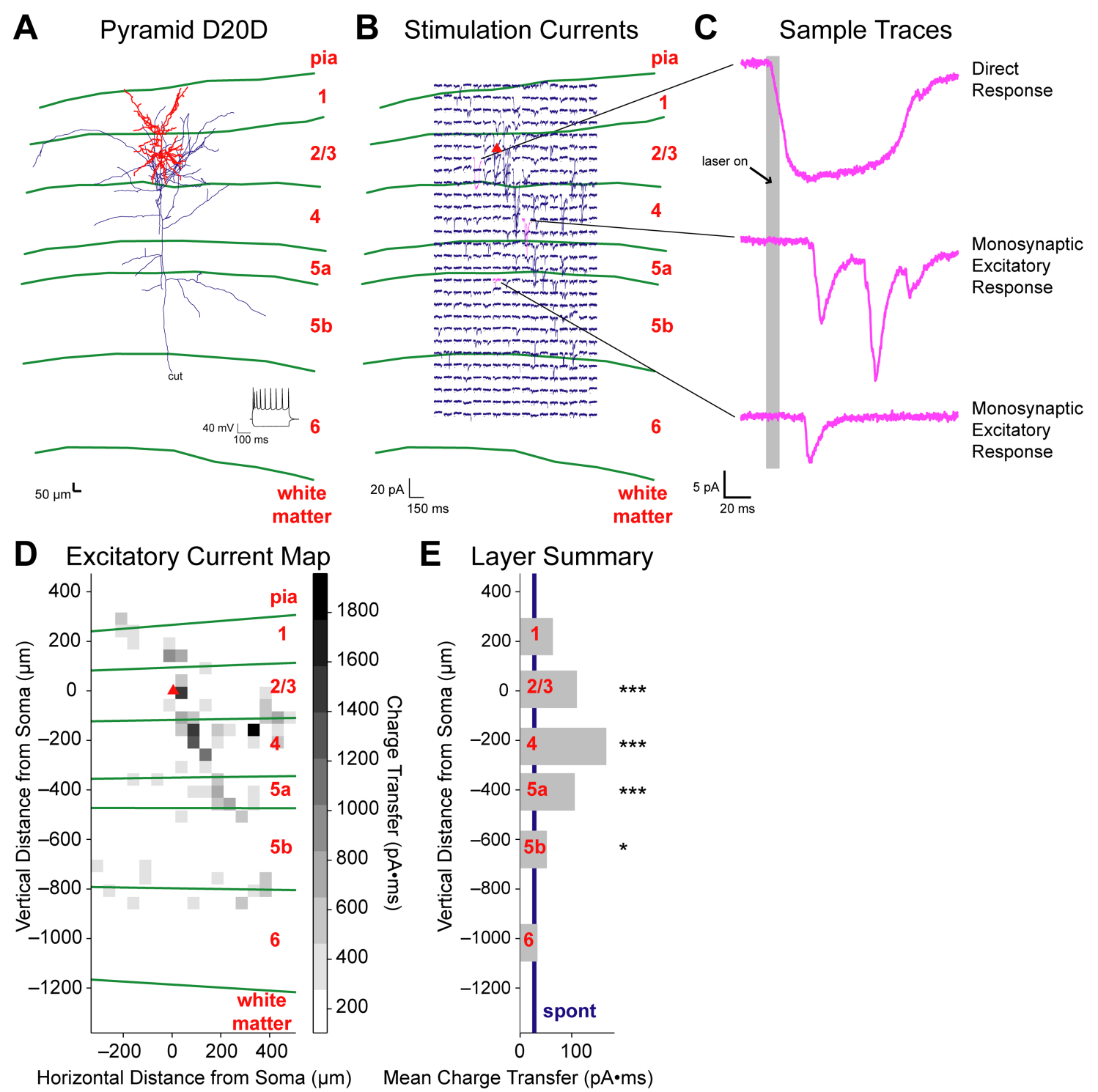

Figure 2. Photostimulation of a layer $2 / 3$ pyramidal neuron. $A$, Drawing of pyramidal neuron dendrites (red) and axons (blue) as well as laminar boundaries (green). Inset shows current-clamp responses to depolarizing and hyperpolarizing currents and regular spiking behavior. $\boldsymbol{B}$, Voltage-clamp current traces (blue) in response to the laser-scanning photostimulation grid reveal both large direct currents seen for stimulation near the soma that are disregarded and EPSCs seen for stimulation elsewhere in the slice. $\boldsymbol{C}$, Expanded versions of sample traces (pink) reveal inward currents. These currents are brought about by (from top to bottom): direct stimulation near the soma, a strong monosynaptic connection and a weaker monosynaptic connection. D, EPSC areas in each trace of $B$ are summed to create a charge transfer measurement for each stimulation site. Grayscale values in all current maps represent deciles from spontaneous activity to maximum stimulated response. $\boldsymbol{E}$, Mean charge transfer for stimulation sites in each cortical layer reveal significant excitatory input from layers $2 / 3,4$, and $5 \mathrm{a}\left({ }^{* * *} p<0.001, \mathrm{ANOVA}\right)$ and $5 \mathrm{~b}\left({ }^{*} p<0.05\right)$.

tic input from excitatory neurons stimulated near their somas. This reasoning follows because spontaneous spiking in the slice is empirically observed to be low, implying that individual excitatory synaptic inputs alone are unlikely to depolarize a postsynaptic neuron sufficiently to induce an action potential. If this condition were not the case, then the current clamp recordings of Figure 1 would show action potentials when the slice was photostimulated away from the patched cell's soma but near the somas of other excitatory neurons monosynaptically projecting to the patched cell. Furthermore, because more action potentials are observed to occur when photostimulation is very close to the soma, a weighted average of postsynaptic activity (see Materials and Methods) allows an improved estimate of the location of presynaptic neuronal somas, as well as an improved ability to quantify relatively weak excitatory input.

Overall, glutamate uncaging by laser scanning photostimula- tion under these experimental conditions has been shown to be capable of revealing the locations of presynaptic neuron somas to an accuracy of $\sim 50-100 \mu \mathrm{m}$, with two major exceptions. First, neurons with extensive apical tufts can also fire action potentials when photostimulated near their tufts (Fig. $1 C, D$ ). Because these tufts are located in layer 1 , photostimulation in layer 1 cannot distinguish between input from cells whose somas are within the layer and cells whose somas are located in another layer but have apical dendrites that tuft in layer 1 . Consequently, layer 1 is excluded from analysis. Few excitatory neurons are located in layer 1 , so this constraint has little influence on maps of excitatory input sources. Second, direct stimulation near the soma of a neuron held in voltage clamp induces a large inward current that can obscure and distort input from monosynaptically connected neurons. Consequently, stimulation sites revealing large direct currents are excluded from analysis. Because these stimulation 
sites are close to the somas of the postsynaptic cells, presynaptic neurons with somas located in this region cannot be adequately resolved with the analysis methods used here.

Preservation of cellular processes from all cortical layers during the slicing process was verified by tracing processes that cross several laminar boundaries. The layer 5B pyramidal neuron shown in Figure $1 D$ has an apical dendrite that was completely located within a single $80 \mu \mathrm{m}$-thick section of the slice, as was half its descending axon. Apical dendrites were always well preserved by the cutting method and descending axons of principal neurons were often preserved within the $400 \mu \mathrm{m}$-thick slices well into the white matter, with the exception that some projecting axons began to bend out of the plane of the slice in layer 6 .

\section{Excitatory input into layer 2/3} pyramidal neurons

Layer 2/3 pyramidal neurons were identified by their large somata and apical dendrites under IR-DIC optics and confirmed anatomically by biocytin fills. An example neuron can be seen in the cell drawing of Figure $2 \mathrm{~A}$ made from a biocytin fill, superimposed onto laminar boundaries drawn from cytochrome oxidase staining. The spiny apical dendrites of these neurons always tufted at the layer 1/layer 2 border. Like superficial pyramidal neurons observed in other primary sensory areas, most axonal projections of these neurons appeared to be local within layer $2 / 3$ with many ramifications ( 35 of 35 adequately filled cells) and additional ramifications in layer 5 (33 of 34 adequately filled cells). Unlike other cortical areas, however, the descending axons of these neurons often sprouted numerous branches within layer 4 (34 of 35 adequately filled cells). Axons from these neurons could occasionally be observed to ramify in layer 6 ( 7 of 30 adequately filled cells) and could often be traced into the white matter ( 13 of 34 adequately filled cells), but in some cases were cut by the slicing procedure before reaching the white matter (19 of 34 adequately filled cells). Depolarizing current pulses injected into the somas of these cells during current clamp revealed regular spiking activity typical of neocortical excitatory cells (Connors and Gutnick, 1990).

Photostimulation of slices in which these neurons were isolated and held in voltage clamp revealed large direct currents for photostimulation sites near the soma, as well as additional monosynaptic EPSCs at other locations in the slice (Fig. 2 B, C). Direct currents distort synaptic currents that result from the same stimulation; therefore, all traces containing direct currents were discarded from further analysis. Excitatory input throughout the slice was quantified by integrating the area under each of the EPSC curves for $150 \mathrm{~ms}$ after laser onset, thereby generating a measure of photostimulation-induced synaptic charge transfer for each site. Charge transfer maps reveal the pattern of excitatory projections within the slice up to a distance of several hundred micrometers away from the postsynaptic cell held under voltage clamp (Fig. 2D). Laminar population summaries were computed from the mean charge transfer for all stimulation sites within each layer. These values were then compared with the mean charge transfer computed during a control interval of $150 \mathrm{~ms}$ during which no photostimulation laser pulse occurred, reflecting spontaneous activity (Fig. 2E). The pyramidal neuron depicted in Figure 2 received significant laminar input from layers $2 / 3,4$ and $5 \mathrm{a}(p<0.001$, Tukey's HSD post hoc corrected ANOVA), as well as layer $5 b(p<0.05)$.

Across the population of pyramidal neurons located throughout layers 2 and 3, excitatory laminar input patterns were assessed in two ways. First, neurons whose mean stimulated charge transfer values for a particular layer were significantly greater than the spontaneous charge transfer values $(p<0.05$, Tukey's HSD post hoc corrected ANOVA) were enumerated. If the number of neurons receiving significant input from any given layer exceeded the number expected by chance (binomial test, success probability, $0.05)$, neurons in that layer were considered to have contributed 

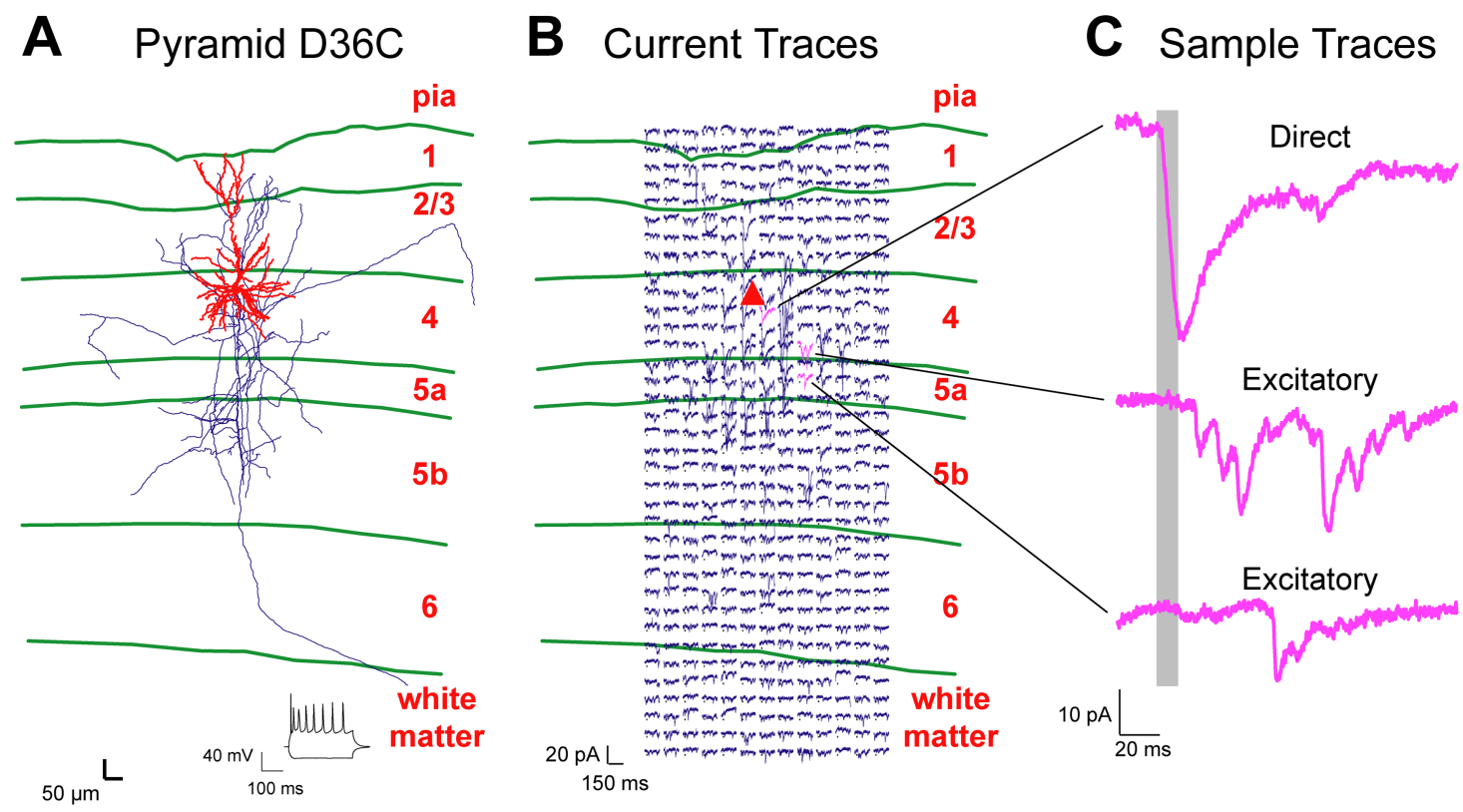

D Excitatory Current Map

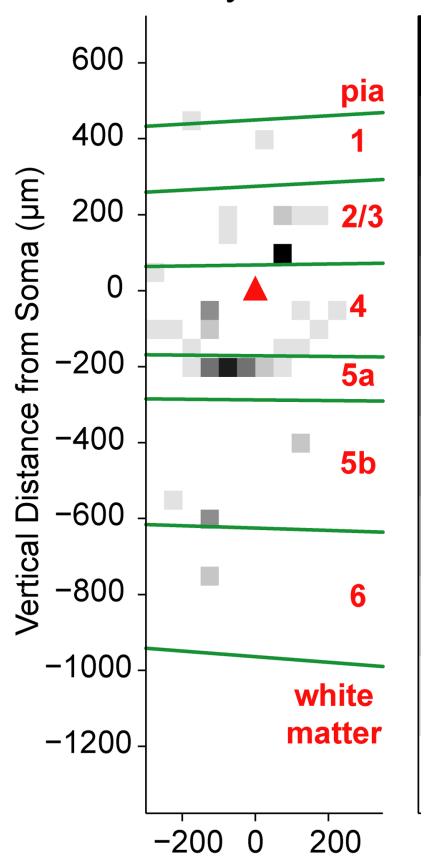

$-2200$

E Layer Summary

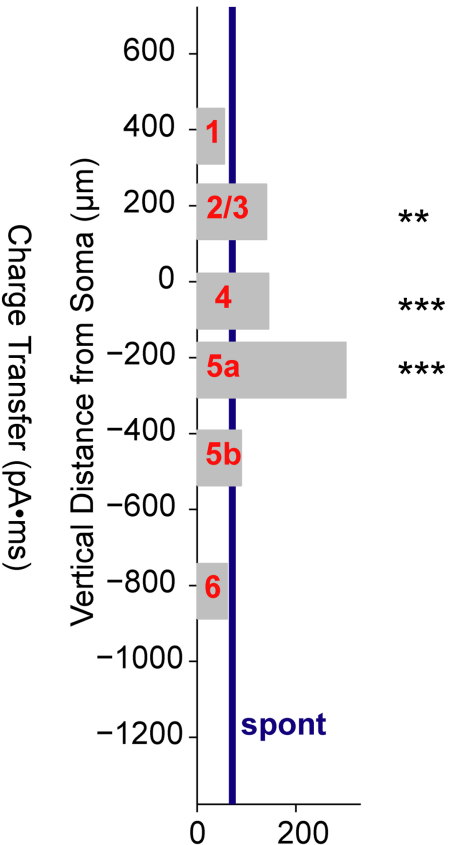

Horizontal Distance from Soma $(\mu \mathrm{m}) \quad$ Mean Charge Transfer $(\mathrm{pA} \cdot \mathrm{ms})$

Figure 5. Photostimulation of a layer 4 pyramidal neuron. $A$, Drawing of pyramidal neuron dendrites (red) and axons (blue) as well as laminar boundaries (green). Inset shows regular spiking behavior. $\boldsymbol{B}$, Voltage-clamp current traces (blue) in response to photostimulation. C, Expanded versions of sample traces (pink) reveal inward currents. D, EPSC areas in each trace of B are summed to create a charge transfer measurement for each stimulation site. Because of the large range of values for this neuron, many sites with low-amplitude stimulated events are mapped to the lowest decile, which yields a white pixel. $\boldsymbol{E}$, Significant excitatory input derives from layers 4 and $5 a\left({ }^{* *} p<0.001\right)$ and layers $2 / 3\left({ }^{* *} p<0.01\right)$.

significantly to the excitatory input of the population of layer $2 / 3$ pyramidal neurons. Second, to compare laminar input directly for neurons across the population, stimulated charge transfer relative to spontaneous charge transfer was converted into $Z$ scores representing a standard normal distribution, and the mean $Z$ score per layer was evaluated relative to $Z=1.96(p=0.05)$. Figure $3 A$ shows that most of the excitatory input into layer $2 / 3$ pyramidal neurons derives from layer 4 (27 of $35, p<0.001$, binomial test; $Z=6.83$ ), followed by layer $2 / 3$ (19 of $35, p<$
$0.001 ; Z=2.53)$ and then by layer 5 a (11 of $35, p<0.001 ; Z=$ 2.22). Significant input was not observed deriving from layer $5 \mathrm{~b}$ ( 4 of $35, p=0.096 ; Z=1.14)$ or layer 6 ( 1 of $35, p=0.83 ; Z=$ $0.33)$.

Whereas layer 2 of A1 appears to receive little thalamocortical input, layer 3 appears to receive as much as layer 4 (Huang and Winer, 2000). This arrangement is unique for primary sensory cortices and raises the possibility that layer 2 neurons participate in different local circuits than layer 3 neurons. Subdividing our 


\section{A Star Pyramid D27B}

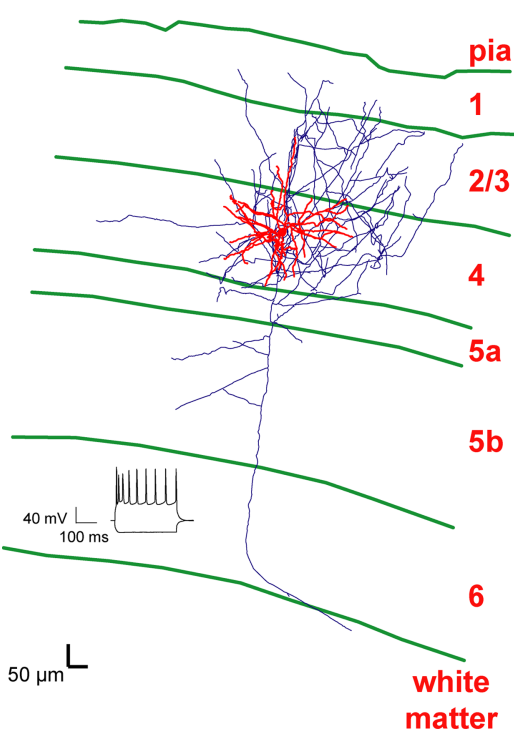

D Excitatory Current Map

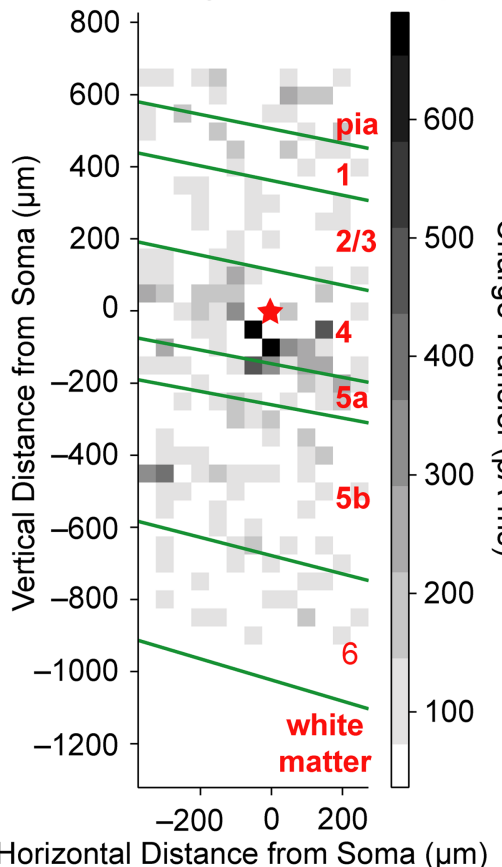

B Current Traces C Sample Traces

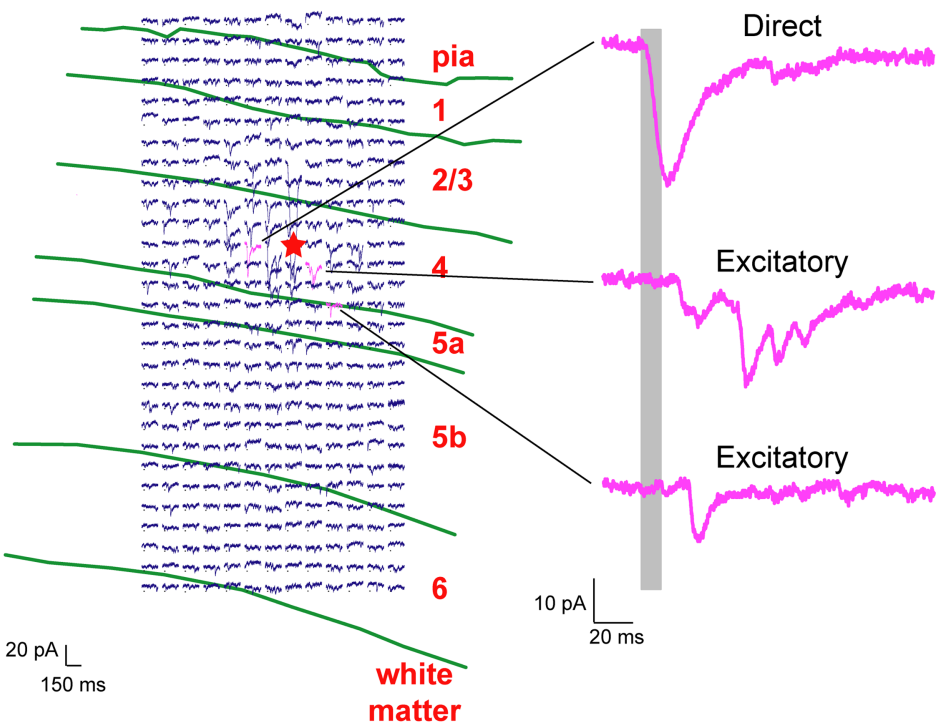

E Layer Summary

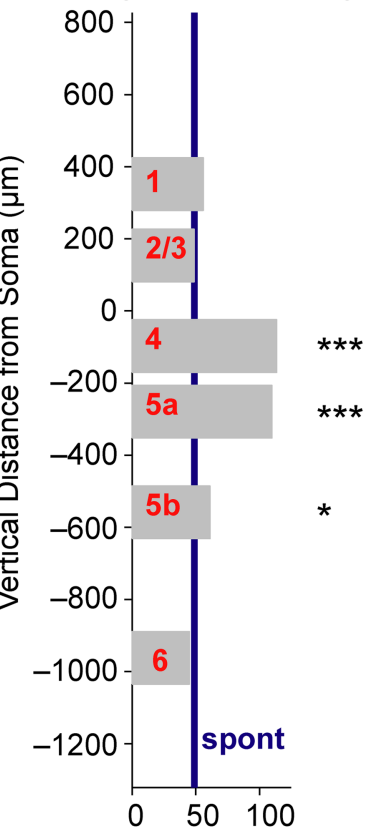

Mean Charge Transfer $(\mathrm{pA} \cdot \mathrm{ms})$

Figure 6. Photostimulation of a layer 4 star pyramid neuron. $A$, Drawing of star pyramid neuron dendrites (red) and axons (blue) as well as laminar boundaries (green). Inset shows regular spiking behavior. $\boldsymbol{B}$, Voltage-clamp current traces (blue) in response to photostimulation. $\boldsymbol{C}$, Expanded versions of sample traces (pink) reveal inward currents. $\boldsymbol{D}$, EPSC areas in each trace of $\boldsymbol{B}$ are summed to create a charge transfer measurement for each stimulation site. $\boldsymbol{E}$, Significant excitatory input derives from layers 4 and $5 \mathrm{a}\left({ }^{* * *} p<0.001\right)$, and layer $5 \mathrm{~b}\left({ }^{*} p<0.05\right)$.

layer $2 / 3$ pyramidal neuron population by vertical position within layers $2 / 3$ (Fig. $3 B$ ) reveals that superficial pyramidal neurons ("layer 2") receive substantially more excitatory layer 4 input than do deeper pyramidal neurons ("layer 3"). Further examination of these laminar input patterns as a function of vertical position within layer $2 / 3$ (Fig. $3 C$ ) reveals that some layer 2 neurons receive more local cortical columnar input overall than do layer 3 neurons, particularly of layer 4 input. This small subpopulation of neurons accounts for the difference in relative proportion of layer 4 excitatory input observed between layer 2 and layer
3 pyramids in Figure $3 B$. This difference is not significant across the population of layer $2 / 3$ pyramidal neurons $(p=0.82$, Wilcoxon rank-sum test of $Z$ scores). The variety in amount of excitatory layer 4 input into layer 2 pyramidal neurons is considerably greater than for layer 3 pyramids $(\sigma=11.9$ for layer 2 pyramids vs $\sigma=4.2$ for layer 3 pyramids), which accounts for the nonsignificance of the difference in $Z$ scores. This distinction may itself reflect different types of excitatory projections between layer 4 to superficial and deep supragranular pyramidal neurons. No systematic excitatory input from layer 6 can be identified 

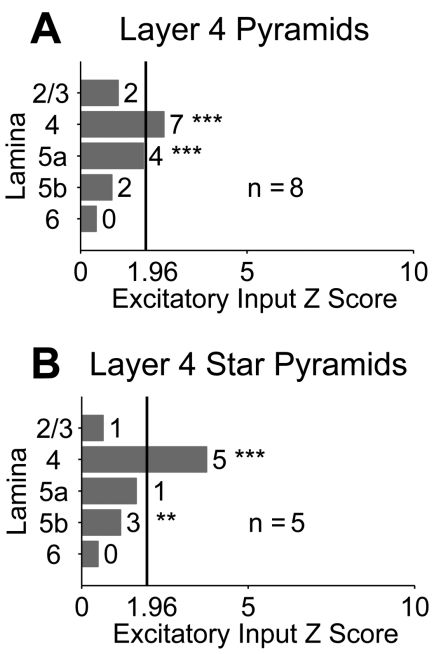

Figure 7. Laminar input summaries for layer 4 spiny neurons. $A, B$, Convention as in Figure $3 A: Z>1.96$ corresponds to $p<0.05 ;{ }^{* *} p<0.001 ;{ }^{* *} p<0.01$. Input from layer 4 also dominates both of these neuronal populations.

across the entire layer $2 / 3$ pyramidal neuron population, as expected from the sparse axonal projection patterns of layer 6 pyramidal neurons to superficial layers in primary sensory cortices (Burkhalter, 1989; Zhang and Deschênes, 1997; Binzegger et al., 2004). Layer 5b excitatory input may be more prevalent in layer 2 pyramidal neurons, but this input is not statistically significant overall and is dwarfed by input from layers 2-5a. Sparser sampling of layer 3 cells relative to layer 2 leaves open the possibility that a denser sampling could reveal more prominent layer 4 input than is implied by the current data.

To evaluate for each layer $2 / 3$ pyramid the relationship between excitatory input deriving from different layers, scatterplots representing laminar input proportions from pairs of layers are plotted in Figure 4. Individual neurons receiving proportionally less layer $2 / 3$ input generally received significantly more layer 4 input, as seen in Figure $4 A$ ( $p<0.001$, regression $F$ test). Despite the significant trend, however, a wide variety of input patterns can be seen for the population, as indicated by the relatively low coefficient of determination $\left(r^{2}=0.33\right)$. Notably, only one neuron received the majority of its excitatory input from layer $2 / 3$ (fraction layer $2 / 3>0.5$ ), whereas a substantial proportion of neurons received their majority input from layer 4 , further demonstrating the dominance of layer 4 excitatory input in this cell population.

A similar but less significant trend can be observed for layer $2 / 3$ input versus layer 5 input in Figure $4 B\left(p<0.02, r^{2}=0.15\right)$. Layer 5 in this case represents layers $5 \mathrm{a}$ and $5 \mathrm{~b}$ combined. No cells of this population received a majority of their excitatory input from layer 5 . When layer 4 and layer 5 inputs are compared in Figure $4 C$, a linear trend is evident, but it is not significant ( $p=$ $0.15, r^{2}=0.06$ ). Therefore, layer $2 / 3$ pyramidal neurons that receive the most layer 4 input tend to receive the least superficial input, but layer 4 input does not predict the degree of deep input.

\section{Excitatory input into layer 4 excitatory neurons}

Although all spiny neurons encountered in layers 2 and 3 exhibited a pyramidal morphology, two distinct morphologies were evident in the population of layer 4 spiny neurons. Layer 4 pyramidal neurons shared dendritic morphological features with layer $2 / 3$ pyramids, including spiny apical dendrites tufting at the layer 1/layer 2 border ( 6 of 6 adequately filled, uncut cells). Ax- onal distribution had a fairly uniform density throughout layers $1-5$ in all these neurons. Descending axons occasionally ramified in layer 6 ( 3 of 7 adequately filled cells) and could typically be traced into the white matter ( 7 of 8 adequately filled, uncut cells). Depolarizing current pulses injected into the somas of these cells during current clamp revealed regular spiking activity. An example neuron of this type can be seen in Figure 5. This neuron received significant excitatory laminar input from layers 4 and $5 \mathrm{a}$ $(p<0.001$, ANOVA), as well as layers $2 / 3(p<0.01)$.

Another type of spiny neuron was encountered just as commonly as pyramidal neurons in layer 4 . These cells possessed an apical dendrite that penetrated into layer 3 but did not reach layer 1 and did not tuft. Most axonal projection density was to layers 2-4 (5 of 5 adequately filled cells) with some ramifications in layer 5 ( 5 of 5 adequately filled cells), rare ramifications in layer 6 ( 1 of 5 adequately filled cells), and common projection into the white matter ( 4 of 4 adequately filled, uncut cells).

In terms of dendritic morphology these neurons resemble somewhat the spiny stellate cells classically observed in thalamocortical recipient layers of primary visual cortex (V1) of multiple species (Lund, 1988). The lack of spiny stellate neurons encountered in this study, however, mirrors previous findings in cat A1 (Smith and Populin, 2001), and provides further evidence that spiny stellate neurons may not participate in auditory cortical circuits. The spiny nonpyramidal neurons in A1 do appear nearly stellate in dendritic morphology, and their axonal projections are heavily biased toward layers $2-4$, as are the spiny stellate neurons of V1 (Binzegger et al., 2004) and primary somatosensory cortex (S1) (Bender et al., 2003). These cells may therefore be assuming the role filled by spiny stellate cells in other primary sensory areas. Nevertheless, they more closely resemble in morphology the classically described "star pyramids" observed in primary sensory cortices of multiple species (Martin and Whitteridge, 1984; Winer, 1984b; Cipolloni and Pandya, 1991; Lübke et al., 2000; Winer and Prieto, 2001; Binzegger et al., 2004; Egger et al., 2008). An example neuron of this type can be seen in Figure 6, and neurons of this type will be referred to as star pyramid neurons. This neuron received significant excitatory laminar input from layers 4 and $5 \mathrm{a}(p<0.001)$, as well as layer $5 \mathrm{~b}(p<0.05)$.

Pyramidal neurons have been observed in thalamocortical recipient layers of other primary sensory areas, but the frequency with which they were encountered in the current study may indicate a unique role for them in auditory cortical processing. Their axonal projections appeared rather uniform throughout layers $1-5$, which is quite distinct from the axonal distributions of star pyramids. Layer 4 pyramidal neurons appeared morphologically similar to layer $2 / 3$ pyramidal neurons, but no star pyramid neurons were encountered in layers $2 / 3$.

Both excitatory cell types encountered in layer 4 received most of their excitatory input from other layer 4 neurons (Fig. 7). Stronger layer 4 input was evident in the star pyramid neurons ( 5 of $5, p<0.001 ; Z=3.76$ ) than in the pyramidal neurons ( 7 of 8 , $p<0.001 ; Z=2.50$ ), although this difference was not statistically significant. For both cell types, less excitatory input was observed from superficial and deep layers compared with the input from layer 4 . Both cell types also appear to receive some excitatory input from layer $2 / 3$, which is an unusual finding. Pyramidal neurons may receive more excitatory input from layer 5 than star pyramids do, but this trend is insufficiently characterized. In terms of laminar excitatory input strength, then, little distinction is evident between these two cell classes.

Scatterplots representing laminar input proportions from 
pairs of layers reveal perhaps the greatest distinction between these two cell types in terms of excitatory input (Fig. 8). The star pyramid neurons exhibit no apparent correlation among the excitatory inputs from different cortical layers. Pyramidal neurons that receive more excitatory input from layer 4, however, appear to receive less excitatory input from layer 5. Because pyramids project densely to layer 5 whereas star pyramids project only sparsely, this difference in correlated input implies that the two classes of excitatory neurons encountered in layer 4 may participate in distinct local microcircuits spanning superficial and deep layers.

\section{Excitatory input into inhibitory cell types}

Two electrophysiological categories of inhibitory interneurons in layers 2-4 were identified by their spiking behavior in response to steps of depolarizing current: fast-spiking and adapting (Connors et al., 1982; Connors and Gutnick, 1990; Agmon and Connors, 1992; Metherate and Aramakis, 1999). Both cell types exhibited smooth, aspiny dendrites with a variety of typically compact dendritic morphologies. Axonal projections were extensive and local with no projections into the white matter. Despite some variety in cell shapes across the population, consistent morphological differences between the two cell types were not immediately apparent, but were not analyzed in detail. Examples of excitatory input maps of fast-spiking and adapting neurons located in layer $2 / 3$ can be seen in Figure 9. These two neurons received significant laminar input from layers $2 / 3$ and $4(p<0.001$, ANOVA $)$ and layer $5 \mathrm{a}(p<0.05$ or $p<0.01)$.

As with the excitatory neurons in layer $2-4$, the inhibitory neurons encountered in these layers received their greatest excitatory input from layer 4 , followed by layers $2 / 3$ and $5 \mathrm{a}$ (Fig. 10). The excitatory input from layers $2 / 3$ is notable for layer 4 neurons (both excitatory and inhibitory) because it implies a direct feedback signal from supragranular neurons to thalamocortical recipient neurons. Deep input was once again relatively weak for these neurons. Layer 4 adapting cells appeared to have weaker input overall than any other cell type, but the small number of such neurons studied makes drawing strong conclusions impossible.

Differences in excitatory input between these two cell populations were again more apparent on examining scatterplots of the excitatory inputs from multiple layers into the same cells (Fig. 11). Only data from layer $2 / 3$ inhibitory neurons are presented because of the small numbers of inhibitory cells isolated in layer 4. Little evidence exists in these data for functionally distinct circuits formed by different classes of inhibitory neurons. This result contrasts with findings obtained previously in rat V1 (Dantzker and Callaway, 2000), implying that the correspon-
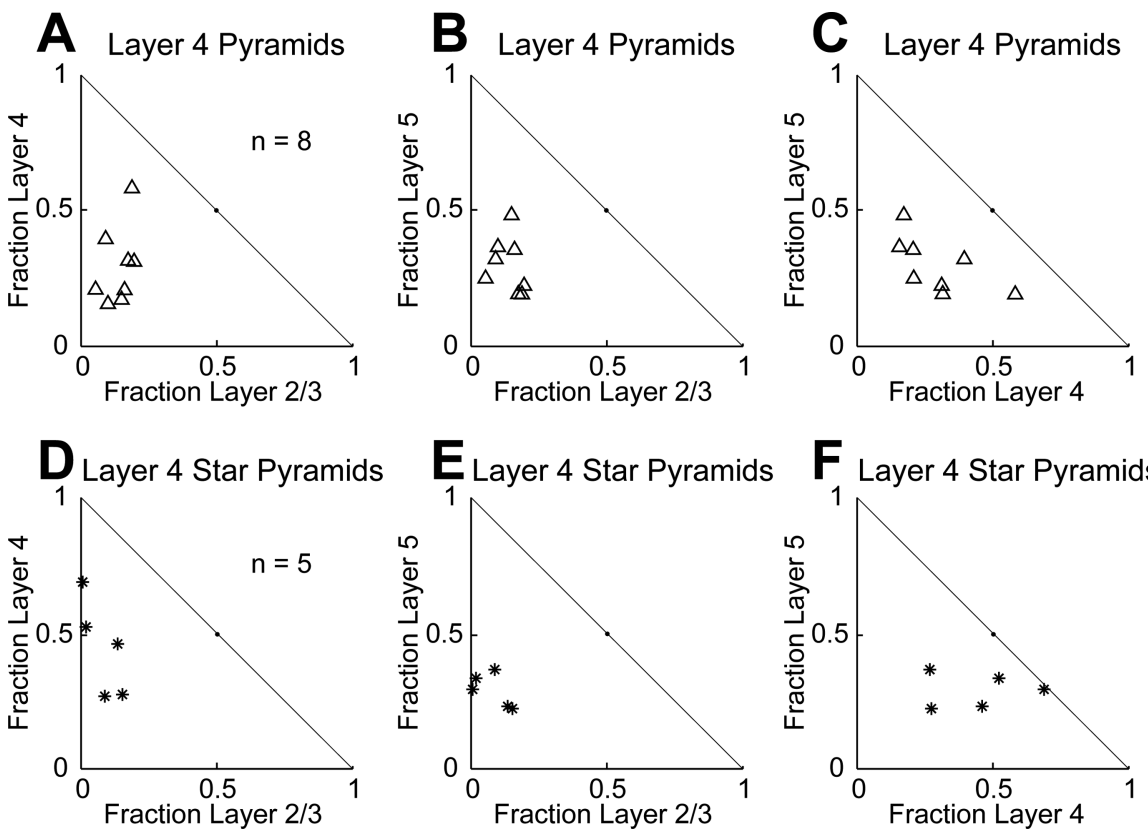

Figure 8. Laminarinput correlations forlayer 4 spiny neurons. $\boldsymbol{A}-\boldsymbol{C}$, Scatterplots showing the pairwise relationships among excitatory implies decreased layer 5 input. $\boldsymbol{D}-\boldsymbol{F}$, Scatterplots showing the pairwise relationships among excitatory layer 4, layer $2 / 3$, and layer 5 input
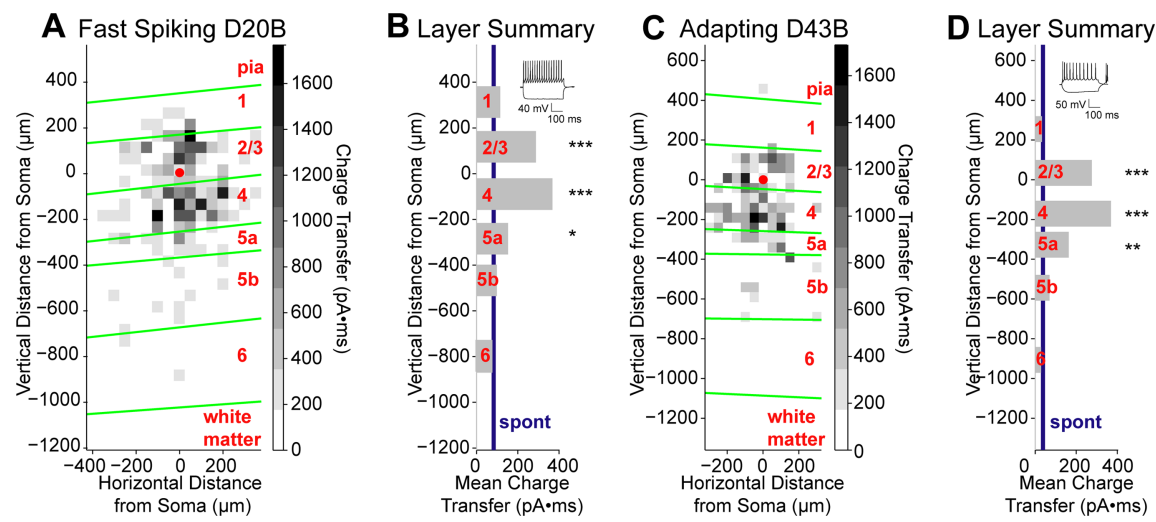

Figure 9. Photostimulation of layer $2 / 3$ interneurons. $\boldsymbol{A}$, Layer $2 / 3$ fast-spiking interneuron charge transfer map. $\boldsymbol{B}$, Layer $2 / 3$ fast-spiking interneuron laminar charge transfer summary revealing significant excitatory input from layers $2 / 3$ and $4\left(^{* * *} p<\right.$ Layer $2 / 3$ adapting interneuron laminar charge transfer summary revealing significant excitatory input from layers $2 / 3$ and 4 $\left({ }^{* * *} p<0.001\right)$ and layer $5 a\left({ }^{* *} p<0.01\right)$.

dence between cell classification and local microcircuitry may not be fixed across cortex or even across primary sensory areas. Alternatively, the subtypes of adapting and FS neurons sampled may differ between these studies. Furthermore, evidence of discrete inhibitory neuronal subpopulations based solely on local microcircuitry that have been discovered in rat V1 was not evident in this data set. This possibility cannot be excluded at present, however, and may require testing of larger samples and more detailed characterization of inhibitory cell types.

\section{Population excitatory laminar input summary}

A complete summary of fractional excitatory laminar input across the entire population of neurons studied can be seen in the mean input proportion plots of Figure 12. All neuron classes found in layers $2 / 3$ tend to have similar excitatory input profiles, 

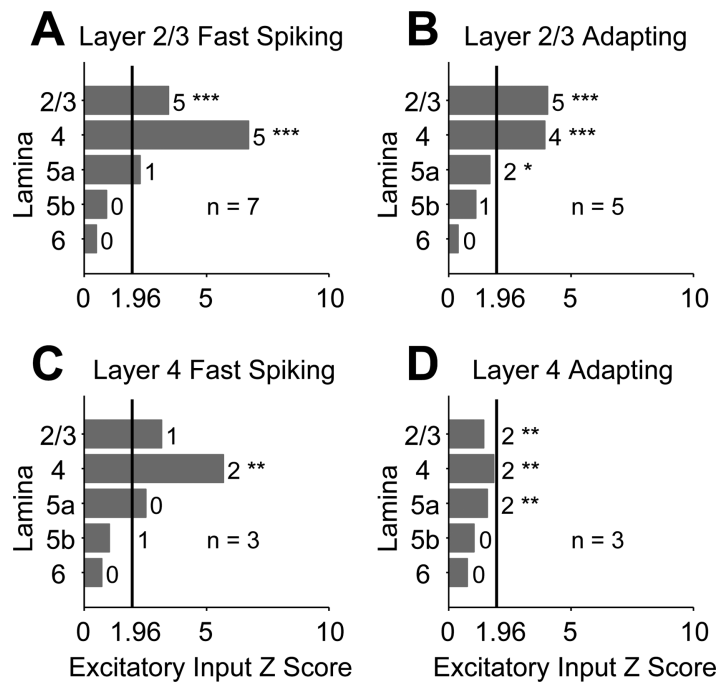

Figure 10. Laminar input summaries for layer $2-4$ aspiny neurons. $A-D$, Convention as in Figure $3 A: Z>1.96$ corresponds to $p<0.05 ;{ }^{* * *} p<0.001 ;{ }^{* *} p<0.01 ;{ }^{*} p<0.05$.

with less than one-third of their input deriving from deep layers. The greatest input variation across this subpopulation is the relative proportion of excitatory input from layers $2 / 3$ and 4 . Layer $2 / 3$ pyramidal neurons, for example, receive over half their excitatory input from layer 4 , adapting inhibitory neurons receive about one-third, and fast-spiking inhibitory neurons are in between.

Substantially more deep excitatory input can be seen in the neurons located in layer 4, up to half of the input in pyramidal and adapting neurons and only slightly less in star pyramids. The greatest distinction between input into pyramidal and star pyramid neurons in layer 4 appears to be the increased layer 4 input and concomitant decreased layer $2 / 3$ input into the star pyramid neurons. The star pyramid neurons are unique among the population studied in that they receive as a population less than onetenth of their excitatory input from layers $2 / 3$, although any input from layer $2 / 3$ to layer 4 cells was unexpected. Layer 4 inhibitory neurons showed a greater variety of input patterns than did superficial inhibitory neurons, with fast spiking neurons receiving substantially more layer 4 input than the adapting neurons. The input patterns for these neuron classes may become more apparent with larger population sizes.

\section{Discussion}

The observations made here demonstrate both similarities and differences between rat A1 and other cortical areas in which local functional circuitry and anatomy have been studied in more detail. Like S1 and V1, A1 is characterized by strong excitatory connections from the classic thalamic recipient layer 4 to layer $2 / 3$, as well as strong recurrent excitation within layer $2 / 3$. Current anatomical and physiological observations provide evidence that the inputs and outputs of layer 4 excitatory neurons in area A1 differ from those in S1 and V1. In particular, layer 2/3 pyramids typically arborize their axons within layer 4 , and these can connect to layer 4 spiny neurons. Furthermore, nearly all layer 4 spiny neurons project out of A1 to extrinsic targets. These observations suggest a difference in the functional role of excitatory layer 4 neurons in A1 compared with S1 or V1.

\section{Photostimulation technique}

Local neocortical circuitry mapping using laser-scanning photostimulation and glutamate uncaging is an established high- throughput, specific technique that reliably reveals monosynaptic projections and the locations of projecting neurons (Callaway and Katz, 1993; Dantzker and Callaway, 2000; Sawatari and Callaway, 2000; Shepherd et al., 2003, 2005; Yoshimura et al., 2005). One limitation of the technique, however, is that input from presynaptic neurons whose somas are located within 100-200 $\mu \mathrm{m}$ of the postsynaptic cell is generally discarded because this signal is obscured by the direct response of the postsynaptic cell. Because of this spatial limitation, photostimulation likely underestimates the strength of monosynaptic excitatory input located within the same cortical lamina as a recipient cell. In the current study, the dominance of layer 4 input into the population of layer 4 neurons is therefore likely to be underestimated, whereas the possibility of greater layer $2 / 3$ input to layer $2 / 3$ neurons cannot be ruled out. Nevertheless, the extent of layer 4 input in all cell types studied strongly implies that most of the neurons in layers 2-4 of rat primary auditory cortex receive the largest proportion of their local excitatory input from layer 4.

The spatial resolution limitations of photostimulation also call for caution in interpreting input assigned to layer $5 \mathrm{a}$, which is generally only $50-100 \mu \mathrm{m}$ thick. It is possible for some of the input assigned to layer 5 a to derive from neurons whose somas and basal dendritic trees are located close to the layer $4 / 5$ border. Clear examples of unambiguous layer 5a input (Fig. 2D), the unique profile of layer $5 \mathrm{a}$ input patterns relative to layer 4 and the observation that layer 4 excitatory neuron dendrites typically respected the layer $4 / 5$ boundary ( 8 of 13 adequately filled cells) all argue toward this potential confound not substantially affecting the overall conclusions drawn from this study.

\section{Unique local connectivity patterns in A1}

Pyramidal neurons were the only excitatory cell type encountered in layer $2 / 3$. These cells exhibited classical pyramidal morphologies with an apical dendrite that tufted at the layer 1/layer 2 border. The most surprising observation about the anatomy of these neurons is the prevalence of axonal arbors in layer 4 . Numerous projections to layer 4 were observed for virtually all layer $2 / 3$ pyramidal neurons. These projections reflect a direct feedback from layer 2/3 to layer 4 that is extremely sparse or absent in other primary sensory areas (Lübke et al., 2003; Binzegger et al., 2004). Perhaps the strongest example of a projection from layer $2 / 3$ pyramids to layer 4 is in mouse S1 (Yabuta et al., 2000), but this projection is present only in the deepest layer 3 pyramidal neurons, whose dendrites extend well into layer 4 , and is absent in more superficial pyramids. Evidence exists that transient projections from layer $2 / 3$ pyramids to layer 4 are present early in development of ferret V1 before being eliminated (Borrell and Callaway, 2002), suggesting that differences in activity between layer $2 / 3$ pyramids in $\mathrm{A} 1$ versus $\mathrm{S} 1$ or $\mathrm{V} 1$ may lead to the differential development of axons in layer 4 . One potential source for such a difference in the activity of layer $2 / 3$ pyramidal neurons is the distinct laminar organization of thalamic input in A1. Layer 3 of rodent and cat A1 receives perhaps as much lemniscal thalamic projection density as does layer 4 (Winer, 1984a; Cruikshank et al., 2002; Winer et al., 2005). Thus, even relatively superficial layer $2 / 3$ pyramids could have basal dendrites receiving direct thalamic input.

In both rodent $\mathrm{S} 1$ and $\mathrm{V} 1$ of species on which data have been published, the main cell population of layer 4 is the spiny stellate. Because their dendrites are confined to layer 4, these neurons can only receive input from cells that have axonal arbors in layer 4 . In these same areas and species, layer $2 / 3$ pyramids do not arborize axons in layer 4 , implying that they do not provide input to the major cell population of layer 4 . In contrast, layer $2 / 3$ input into layer 4 appears to be relatively common in A1, which could imply 
the existence of a fast intracolumnar feedback mechanism. Such connections may play an important role in rapidly creating auditory stimulus specificity in cortical neurons (Kaur et al., 2004; Liu et al., 2007) or generating specialized receptive field structures not found in other sensory areas, such as intensity tuning (Wehr and Zador, 2003; Tan et al., 2007).

Layer 4 excitatory neurons exhibited two morphologies: pyramidal and star pyramidal. Star pyramids do not appear to be a common cell type in other sensory areas but have been observed previously in auditory cortex (Winer, 1984b; Cipolloni and Pandya, 1991; Fitzpatrick and Henson, 1994; Winer and Prieto, 2001). Notably absent in rat layer 4 are spiny stellate cells, which are well known in thalamocortical recipient layers of $\mathrm{V} 1$ and appear to represent the majority of excitatory cells in rat barrel cortex layer 4 (Lübke et al., 2000). Spiny stellate neurons have been reported in cat A1 (Winer, 1984a), but this observation has also been disputed (Smith and Populin, 2001). Layer 4 in mustached bat A1 contains spiny nonpyramidal neurons with dendrites extending into layer 3 (Fitzpatrick and Henson, 1994), implying that this cellular morphology may generalize to multiple species. The prevalence of pyramidal neurons in layer 4 of rat A1 is also notable, as this cell type appears to be relatively rare in thalamocortical recipient layers of other primary sensory areas (Lund, 1988; Binzegger et al., 2004).

Star pyramids and pyramidal neurons in layer 4 of rat A1 both represent projection neurons, as do layer $2 / 3$ pyramids. Coupled with the direct excitatory feedback from layer $2 / 3$ to layer 4 , this finding implies that these cells provide a more direct and possibly more rapid relay between cortical input and output than layer 4 neurons in other primary sensory cortical areas, which tend to have exclusively local projections (Martin and Whitteridge, 1984; Lübke et al., 2000). All axons entering the white matter from layer 2-4 cells bent dorsally, and some could be followed for hundreds of micrometers within the same section. The targets of these projections are therefore not likely to be another frequency region of A1 or another core cortical area, all of which would be located in anterior or posterior directions or possibly ventrally (Doron et al., 2002; Rutkowski et al., 2003). Neither are auditory subcortical structures likely to be the targets, as cells projecting to these areas are located in deep layers (Doucet et al., 2002, 2003). Other subcortical nuclei, nonprimary auditory cortical areas, nonauditory cortical areas or contralateral auditory cortical areas (Jacobson and Trojanowski, 1974) remain potential targets for these neurons.

\section{Input patterns across layers}

All the neuron types examined in layers 2-4 of rat A1 derived most of their excitatory input from layer 4 . As expected from the sparse axonal projection patterns of layer 6 pyramidal neurons to superficial layers in primary sensory cortices (Burkhalter, 1989; Zhang and Deschênes, 1997; Binzegger et al., 2004), substantial excitatory input into layer 2-4 neurons deriving from layer 6 was not apparent. Such weak layer 6 input mirrors findings from previous studies making use of photostimulation (Dantzker and Callaway, 2000; Shepherd et al., 2003, 2005). Some weak excitatory input into all cell types was
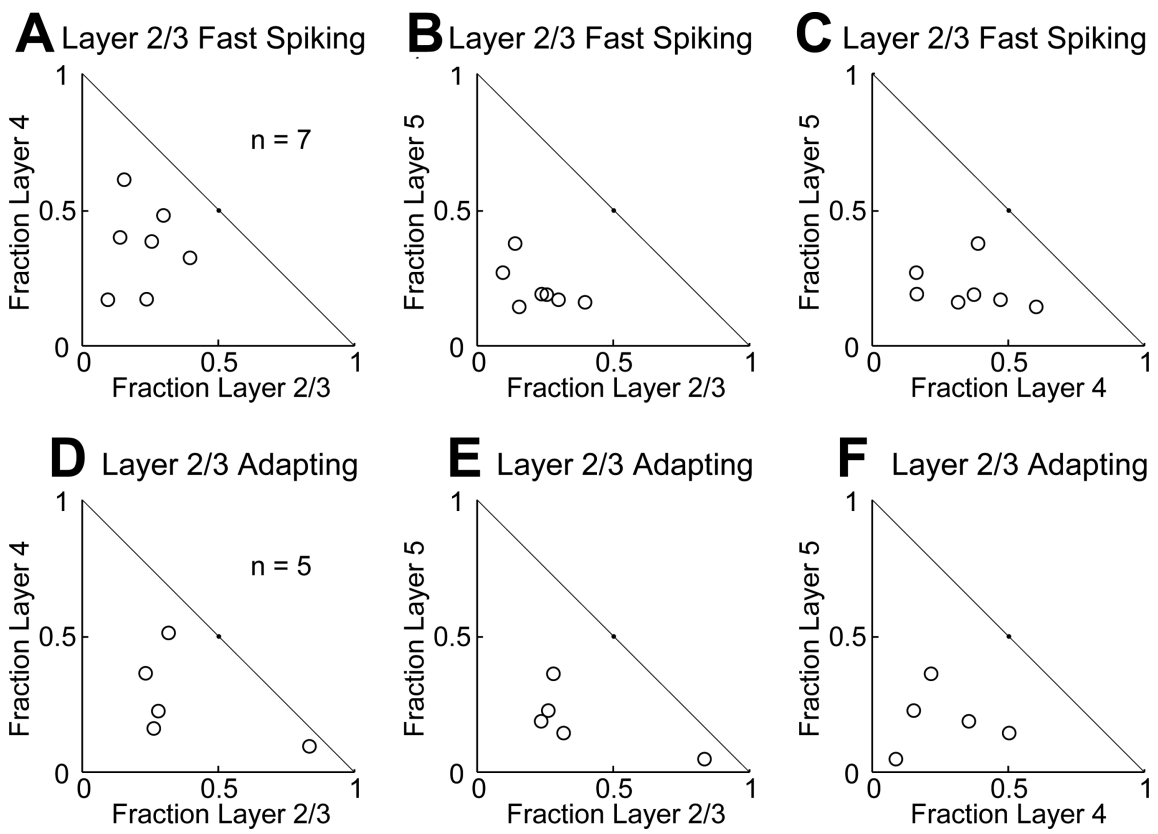

Figure 11. Laminar input correlations for layer 2/3 aspiny neurons. $\boldsymbol{A}-\boldsymbol{C}$, Scatterplots showing the pairwise relationships relationships among excitatory layer 4 , layer $2 / 3$, and layer 5 input for layer 2/3 adapting neurons. Both of these neuronal populations appear to have a range of possible excitatory input patterns.

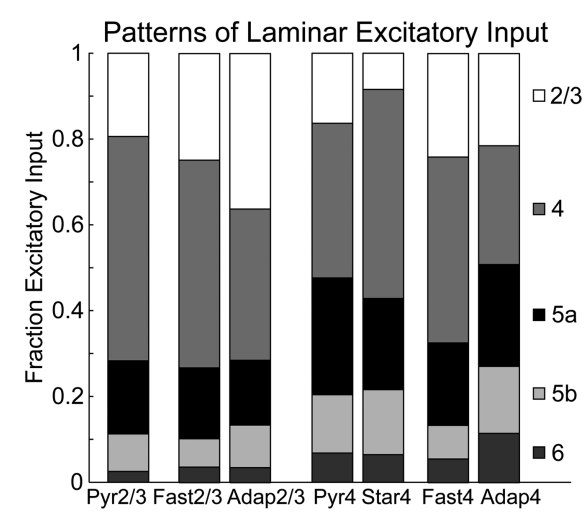

Figure 12. Laminar input proportions for all cell types. The mean fractional excitatory input from each of the laminar subdivisions demonstrates that layer 4 represents the single greatest laminar input into each neuronal type, and layer 4 neurons receive more deep input than do layer 2/3 neurons.

evident originating from layer $5 b$, but none that achieved significance except in layer 4 star pyramid neurons, and there with only one of the two statistical measures used.

As a population, layer $2 / 3$ excitatory and inhibitory neurons derived their second greatest excitatory input locally from layer $2 / 3$. Layer 4 neurons, however, received somewhat less layer $2 / 3$ input overall and considerably more deep input from layers 5 and 6 . Layer $2 / 3$ pyramidal neurons demonstrated inverse input relationships between layers $2 / 3$ and 4 and between layers $2 / 3$ and 5 , but no such relationship between layers 4 and 5 . Layer 4 excitatory projections to layer $2 / 3$ pyramidal neurons therefore appear to create specific microcircuits along a continuum of projection strength, but layer 5 input is fairly constant across this continuum. This putative circuitry structure is reminiscent of the finescale layer $2 / 3$ microcircuits in rat $\mathrm{V} 1$ that all receive common layer 5 input (Yoshimura et al., 2005). 
Within layer $2 / 3$, superficial pyramidal neurons appear to receive more layer 4 excitatory input than deeper neurons, implying that pyramidal neurons take part in different local circuits as a function of depth. As noted above, even layer 2 pyramidal neurons could potentially receive some direct lemniscal thalamic input in A1, but the strength of this input would be expected to be less than for pyramidal neurons located in layer 3. One reasonable possibility for this finding is that extra layer 4 input provides layer 2 pyramids with a stronger disynaptic thalamic excitation to balance the weaker direct thalamic input, whereas the balance of direct versus disynaptic thalamic influence is stronger for layer 3 pyramids (Winer, 1984a, 2005; Cruikshank et al., 2002).

\section{References}

Agmon A, Connors BW (1992) Correlation between intrinsic firing patterns and thalamocortical synaptic responses of neurons in mouse barrel cortex. J Neurosci 12:319-329.

Atzori M, Lei S, Evans DI, Kanold PO, Phillips-Tansey E, McIntyre O, McBain CJ (2001) Differential synaptic processing separates stationary from transient inputs to the auditory cortex. Nat Neurosci 4:1230-1237.

Barbour DL, Callaway EM (2004) Excitatory connections in superficial rat primary auditory cortex. Soc Neurosci Abs 30:987.12.

Bender KJ, Rangel J, Feldman DE (2003) Development of columnar topography in the excitatory layer 4 to layer $2 / 3$ projection in rat barrel cortex. J Neurosci 23:8759-8770.

Binzegger T, Douglas RJ, Martin KA (2004) A quantitative map of the circuit of cat primary visual cortex. J Neurosci 24:8441-8453.

Borrell V, Callaway EM (2002) Reorganization of exuberant axonal arbors contributes to the development of laminar specificity in ferret visual cortex. J Neurosci 22:6682-6695.

Burkhalter A (1989) Intrinsic connections of rat primary visual cortex: laminar organization of axonal projections. J Comp Neurol 279:171-186.

Callaway EM (2002) Cell type specificity of local cortical connections. J Neurocytol 31:231-237.

Callaway EM, Katz LC (1993) Photostimulation using caged glutamate reveals functional circuitry in living brain slices. Proc Natl Acad Sci U S A 90:7661-7665.

Cipolloni PB, Pandya DN (1991) Golgi, histochemical, and immunocytochemical analyses of the neurons of auditory-related cortices of the rhesus monkey. Exp Neurol 114:104-122.

Connors BW, Gutnick MJ (1990) Intrinsic firing patterns of diverse neocortical neurons. Trends Neurosci 13:99-104.

Connors BW, Gutnick MJ, Prince DA (1982) Electrophysiological properties of neocortical neurons in vitro. J Neurophysiol 48:1302-1320.

Cruikshank SJ, Rose HJ, Metherate R (2002) Auditory thalamocortical synaptic transmission in vitro. J Neurophysiol 87:361-384.

Dantzker JL, Callaway EM (2000) Laminar sources of synaptic input to cortical inhibitory interneurons and pyramidal neurons. Nat Neurosci 3:701-707.

Doron NN, Ledoux JE, Semple MN (2002) Redefining the tonotopic core of rat auditory cortex: physiological evidence for a posterior field. J Comp Neurol 453:345-360.

Doucet JR, Rose L, Ryugo DK (2002) The cellular origin of corticofugal projections to the superior olivary complex in the rat. Brain Res 925:28-41.

Doucet JR, Molavi DL, Ryugo DK (2003) The source of corticocollicular and corticobulbar projections in area Tel of the rat. Exp Brain Res 153:461-466.

Egger V, Nevian T, Bruno RM (2008) Subcolumnar dendritic and axonal organization of spiny stellate and star pyramid neurons within a barrel in rat somatosensory cortex. Cereb Cortex 18:876-889.

Fitzpatrick DC, Henson OW Jr (1994) Cell types in the mustached bat auditory cortex. Brain Behav Evol 43:79-91.

Huang CL, Winer JA (2000) Auditory thalamocortical projections in the cat: laminar and areal patterns of input. J Comp Neurol 427:302-331.

Jacobson S, Trojanowski JQ (1974) The cells of origin of the corpus callosum in rat, cat and rhesus monkey. Brain Res 74:149-155.

Jin X, Prince DA, Huguenard JR (2006) Enhanced excitatory synaptic connectivity in layer $\mathrm{v}$ pyramidal neurons of chronically injured epileptogenic neocortex in rats. J Neurosci 26:4891-4900.
Katz LC, Dalva MB (1994) Scanning laser photostimulation: a new approach for analyzing brain circuits. J Neurosci Methods 54:205-218.

Kaur S, Lazar R, Metherate R (2004) Intracortical pathways determine breadth of subthreshold frequency receptive fields in primary auditory cortex. J Neurophysiol 91:2551-2567.

Kim U, Ebner FF (1999) Barrels and septa: separate circuits in rat barrels field cortex. J Comp Neurol 408:489-505.

Koralek KA, Jensen KF, Killackey HP (1988) Evidence for two complementary patterns of thalamic input to the rat somatosensory cortex. Brain Res 463:346-351.

Liu BH, Wu GK, Arbuckle R, Tao HW, Zhang LI (2007) Defining cortical frequency tuning with recurrent excitatory circuitry. Nat Neurosci 10:1594-1600.

Lübke J, Egger V, Sakmann B, Feldmeyer D (2000) Columnar organization of dendrites and axons of single and synaptically coupled excitatory spiny neurons in layer 4 of the rat barrel cortex. J Neurosci 20:5300-5311.

Lübke J, Roth A, Feldmeyer D, Sakmann B (2003) Morphometric analysis of the columnar innervation domain of neurons connecting layer 4 and layer 2/3 of juvenile rat barrel cortex. Cereb Cortex 13:1051-1063.

Lund JS (1988) Anatomical organization of macaque monkey striate visual cortex. Annu Rev Neurosci 11:253-288.

Martin KA, Whitteridge D (1984) Form, function and intracortical projections of spiny neurones in the striate visual cortex of the cat. J Physiol 353:463-504.

Metherate R, Aramakis VB (1999) Intrinsic electrophysiology of neurons in thalamorecipient layers of developing rat auditory cortex. Dev Brain Res 115:131-144.

Roger M, Arnault P (1989) Anatomical study of the connections of the primary auditory area in the rat. J Comp Neurol 287:339-356.

Rutkowski RG, Miasnikov AA, Weinberger NM (2003) Characterisation of multiple physiological fields within the anatomical core of rat auditory cortex. Hear Res 181:116-130.

Sawatari A, Callaway EM (2000) Diversity and cell type specificity of local excitatory connections to neurons in layer $3 \mathrm{~B}$ of monkey primary visual cortex. Neuron 25:459-471.

Shepherd GM, Pologruto TA, Svoboda K (2003) Circuit analysis of experience-dependent plasticity in the developing rat barrel cortex. Neuron 38:277-289.

Shepherd GM, Stepanyants A, Bureau I, Chklovskii D, Svoboda K (2005) Geometric and functional organization of cortical circuits. Nat Neurosci 8:782-790.

Smith PH, Populin LC (2001) Fundamental differences between the thalamocortical recipient layers of the cat auditory and visual cortices. J Comp Neurol 436:508-519.

Tan AY, Atencio CA, Polley DB, Merzenich MM, Schreiner CE (2007) Unbalanced synaptic inhibition can create intensity-tuned auditory cortex neurons. Neuroscience 146:449-462.

Wallace MN, Palmer AR (2008) Laminar differences in the response properties of cells in the primary auditory cortex. Exp Brain Res 184:179-191.

Wehr M, Zador AM (2003) Balanced inhibition underlies tuning and sharpens spike timing in auditory cortex. Nature 426:442-446.

Winer JA (1984a) Anatomy of layer IV in cat primary auditory cortex (AI). J Comp Neurol 224:535-567.

Winer JA (1984b) The pyramidal neurons in layer III of cat primary auditory cortex (AI). J Comp Neurol 229:476-496.

Winer JA, Prieto JJ (2001) Layer V in cat primary auditory cortex (AI): cellular architecture and identification of projection neurons. J Comp Neurol 434:379-412.

Winer JA, Miller LM, Lee CC, Schreiner CE (2005) Auditory thalamocortical transformation: structure and function. Trends Neurosci 28:255-263.

Yabuta NH, Callaway EM (1998) Cytochrome-oxidase blobs and intrinsic horizontal connections of layer $2 / 3$ pyramidal neurons in primate $\mathrm{V} 1$. Vis Neurosci 15:1007-1027.

Yabuta NH, Butler AK, Callaway EM (2000) Laminar specificity of local circuits in barrel cortex of ephrin-A5 knockout mice. J Neurosci 20:RC88.

Yoshimura Y, Dantzker JL, Callaway EM (2005) Excitatory cortical neurons form fine-scale functional networks. Nature 433:868-873.

Zhang ZW, Deschênes M (1997) Intracortical axonal projections of lamina VI cells of the primary somatosensory cortex in the rat: a single-cell labeling study. J Neurosci 17:6365-6379. 